\title{
Engagement of Care for People Affected by the Covid-19 Pandemic: A Research Perspective
}

\section{Changsuphan $S^{*}$}

Sukhothai Thammathirat Open University, School of Nursing, Thailand

*Corresponding author: Supattra Changsuphan, Lecturer in School of Nursing, Sukhothai, Thammathirat Open University, Thailand, Email: supattra.map@stou.ac.th

\section{Editorial}

Volume 4 Issue 3

Received Date: June 19, 2021

Published Date: June 24, 2021

DOI: $10.23880 /$ jqhe- 16000228

\section{Editorial}

The disruption caused by the current Covid-19 pandemic has caused various types of impacts on the multiple dimensions of society and human life. These have required a significant adjustment to normal life, and we have now settled into a 'new normal' life which includes social distancing, wearing face masks, changes to working and learning lifestyles, and financial effects while trying to maintain a stable and safe existence during this highly disruptive period. Engagement in living means that individuals are expected to agree when to seek any care or support, dispensed by planners and providers, to meet their healthcare management needs.

Although the government or administration of each country is working diligently to cope with huge challenges in the context of the ongoing health crisis, they are facing serious limitations and difficulties. As a result, many people are frustrated and suffering, feel unsettled in their uncertain and unpredictable life, and sense they are not fully engaging in or enjoying life. Indeed, there is a great deal of variability among people's optimal lifestyles. Some of their concerns and basic differences include the following:

- Cultural backgrounds and beliefs,

- Varied educational backgrounds,

- Physical, health problems and conditions,

- Differences in psychological states (mentality, support needs, and ability to cope),

- Highly valued and marginalized groups of people,

- Society, environment, financial support, and technology.

The ongoing effects of Covid-19 have affected the lifestyles of people who now live a 'new normal' life. Healthcare teams have created a new culture of care whereby the number of protocols or interventions provided for patients by healthcare institutes and communities has promoted knowledge about healthy lifestyles, psychological responses, and suffering etc. For example, healthcare teams have challenged the transition to online healthcare services to maintain standards of care and to conduct research. From telephone and online communication services, we must choose a simple technology device that provides prompt communication (teletherapy) whereby psychiatric treatment is delivered online, such as via video conferencing. This should be used to communicate, record, report, and evaluate caring programs among clients and healthcare teams. Even though another device may be uncomfortable, access to the internet is not limited to a computer desktop.

Although healthcare professionals and researchers have tried to create innovative care systems during Covid-19, some people/clients are limited by applications, processes, and platforms. A client's low income may cause limited access; senior citizens may not be able to use or understand smartphones and applications; and remote areas may be deprived of modern technology, such as broadband. So, clients/people are challenging their engagement during this Covid-19 age.

Healthcare workers and researchers not only focus on providing effective programs of care, but are also aware of the background of their clients in the limited context of:

- Cultural and educational backgrounds and beliefs,

- Physical problems,

- The need for psychological support,

- Valuable groups of people,

- Social needs,

- Financial support needs,

- The environment, and 
- Technology.

Accordingly, a critical factor that may interrupt client engagement is their perception of roles and responses. Healthcare workers and researchers should closely observe and have a deep understanding of clients/people to afford them an effective and evaluated program of care.

\section{Conclusion}

Healthcare professionals and researchers have provided effective care programs appropriate for clients and their social conditions during each phase of the Covid-19 crisis. It is essential to consider these programs to evaluate and account for their ability to engage clients' needs. 